\title{
Research on the Prediction Algorithm of the Arrival Time of Campus Bus
}

\author{
MENG Lei \\ Jilin University \\ Changchun, China
}

\author{
LI Peiying, WANG Junbao, ZHOU Zhiyong \\ Jilin University \\ Changchun, China
}

\begin{abstract}
The main content of this paper is the prediction algorithm of the time that the campus bus needs to arrive at the position of the passenger. The prediction algorithm uses a method of piecewise prediction. According to the historical average velocity data of each section of route, the residence time of the campus bus on each section is predicted, and then the total time is predicted. The historical average velocity data is updated by the exponential smoothing method, which makes the new data account for a higher proportion of the forecast.
\end{abstract}

Keywords - arrival time prediction; Intelligent Transportation; exponential smoothing method; historical average model

\section{INTRODUCTION}

The operation of the campus bus is a great convenience for students to go out. However through the field survey, we found that many students spending too much time waiting for campus bus and hoping to reduce the waiting time. Intelligent transportation is an appropriate method to solve this problem. At present, the bus arrival time prediction has been widely used and obtained remarkable effect. Therefore, it is feasible to reduce the waiting time by predicting the time required for the arrival of the campus bus. At present, the bus arrival time prediction algorithm has the following 8 models $^{[1]}$ : (1) historical average model (2) time series model (3) regression model (4) Nonparametric regression model (5) artificial neural network model (6) Kalman Filtering Model (7) combination model (8) other models. Based on the characteristics of the campus bus operating environment, this paper established the prediction model that according to the historical average data to predict the arrival time of campus bus.

\section{Models AND AlgORITHMS}

\section{A. Analysis on Running Characteristics of Campus Bus}

The running of the campus bus has the following characteristics:

- Less traffic jams As the campus bus running in the campus, and avoiding busy areas, it meets with less traffic jams.

- Departure time is not fixed There is no fixed timetable for the campus bus, the departure time is decided by the driver. Drivers are to try to make the bus filled with people, so the number of the people waiting for campus bus more, the campus bus starts faster.
- Students can get on the bus at any location within the route There is no station on campus, students can get on the bus at any location within the route; while the city bus has fixed bus stops, passengers can only get on the city bus at some fixed locations.

- Small time correlation Urban transportation systems often have a lot of time correlation, and there is a big difference between rush hour and noon, working days and weekends. Due to less traffic jams on campus, the campus bus time correlation is small.

\section{B. Travel Time Prediction}

This section will discuss the prediction of the time required for a campus bus to arrive at a passenger's position. This will be based on the real-time GPS positioning data and the average speed of each road. The speed of a campus bus on the whole course is variable, but in a short route, the speed will change in a small range. After dividing the route, as shown in the following figure.

$\begin{array}{llll}\text { section } i & \text { section } i+1 & \ldots \ldots & \text { section } j \\ \text { campus bus } & & & \text { passenger }\end{array}$

Fig.1. Dividing the Route

So divide the time needed to reach the passengers:

- The time required for going through the remaining distance of the section $i$.

- The time required for going through the distance between section $i$ and section $j$.

- The time required for going through the distance between the beginning of section $j$ and the passenger's position

\section{B.1 The Time Required for Passing the Remaining Distance of the Section $i$}

By predicting the average speed of the campus bus going through the remaining distance of the section $i$, the time required is predicted. The formula of velocity prediction: 


$$
\begin{gathered}
P=\sigma R+(1-a) p \\
a=\frac{i}{2}
\end{gathered}
$$

Which:

$P$ is the predicted average speed of the current location of the campus bus to the end of this section;

$V$ is the historical average speed of the section $i$, which acts as a historical reference;

$l$ on behalf of the distance that the campus bus has gone through of the section $i$;

$L$ for the length of the section $i$.

\section{B.2 The Time Required for Passing the Distance between Section $i$ and Section $j$}

This part of the time is inferred from historical data. Set $v_{x}$ to the historical average speed of the section $x$. The forecast time is:

$$
t_{x}=\frac{z}{v_{x}}
$$

Which:

$t_{x}$ is the forecast time through the section;

$L$ is the length of the section;

$v_{x}$ is the historical average speed of $x$.

B.3 The Time Required for Passing the Distance between the Beginning of Section $j$ and the Passenger's Position

This part of the time is inferred from historical data. Set $v_{j}$ to the historical average speed of the section $j$. Ignore the time spent on the brake on the campus bus. The time required from the starting position of the section $j$ to the passenger position is:

$$
t_{j}=\frac{i}{v_{l}}
$$

Which:

Section $A$ represents the section from the start position of the section $j$ to the passenger position;

$t_{j}$ is the forecast time required for passing section $A$;

$l$ is the length of section $A$;

$v_{j}$ is the historical average speed of $j$.

\section{B.4 Consideration of Time Spent on Passenger Getting On}

If there are other passengers to get on between the campus bus and passenger $a$, the passenger $a$ 's waiting time will increase. Set $x$ to the section that the passenger belongs to; $v_{x}$ to the historical average speed of section $x$; assume that the brake acceleration and start acceleration of campus bus are equal and constant, which is set to $A$; the time required for the passenger getting on is $T$, which is a constant. So the additional time for passenger getting on is:

$$
\xi_{\text {wp }}=\frac{k_{i}}{\lambda}+T
$$

\section{B.5 Update of Section Historical Average Speed}

In order to better predict the time of the campus bus passing through the section, the historical average speed of the section needs to be updated in real time. According to the GPS position information of the campus bus, when the campus bus into the section, record the current time $\iota_{1}$; when the campus bus to leave the section, record the current time $t_{2} ;\left(t_{2}-t_{1}\right)$ is the actual time of the campus bus passing through the section. The updated method uses exponential smoothing method:

$$
F_{t}=a y_{t}+(1-x) p_{t-1}
$$

Which:

$V_{z}$ is the new historical average speed of this section;

$\nabla_{t-1}$ is the previous historical average speed of this section; $y_{t}$ is the actual time of the passage of this section this time; $a$ is a smoothing constant, this paper takes $a$ as 0.35 .

If there are passengers need to get on the bus in this section, then the actual time which the campus bus needs to pass the section will be longer. So the data can be abandoned for the accuracy of historical data.

\section{EXPERIMENT}

In this paper, we design the experiments for the time prediction algorithm, and verify the accuracy of the algorithm.

\section{A. Experimental Environment}

Algorithm written in the PHP language is deployed in the apache server. Server host configuration: single core CPU, 2GB memory, 40GB hard disk. The operating system is Windows Server 2012.The phone model of client are onePlus A3010 and 2amsung SM - A9000. OnePlus A3010 installs the client for drivers, and SAMSUNG SM-A9000 installs the client for students. In addition, android version is 6.0 or above. A mobile $4 \mathrm{~g}$ network is adopted for communication.

\section{B. The Experimental Steps}

(1) A sits in campus bus, starting the client for drivers.

(2) $B$ chooses an arbitrary position on campus bus route, starting the client for students.

(3) $B$ calls the campus bus. 
(4) After the campus bus starting, $B$ selects 2 or 3 different positions between campus bus and $B$ randomly. When the bus arrives at position $x_{i}$, the student client requests predicted arrival time, and records the current time $T_{i}$.

(5) When the bus arrives at the position of $B, B$ records the current time $\boldsymbol{T}_{\text {end }}$.

(6) Calculate the actual arrival time according $\left(T_{\text {mad }}-T_{h}\right)$.

\section{The Experimental Results}

According to the above steps 14 sets of data are obtained, arranged by the order of the test. As shown in the following table.

TABLE1. The Experimental Results

\begin{tabular}{cc}
\hline $\begin{array}{c}\text { Predicted Arrival Time } \\
\text { (Unit:s) }\end{array}$ & $\begin{array}{c}\text { Actual Arrival Time } \\
\text { (Unit:s) }\end{array}$ \\
\hline 114 & 104 \\
21 & 36 \\
86 & 94 \\
69 & 60 \\
28 & 44 \\
123 & 115 \\
63 & 72 \\
22 & 25 \\
116 & 120 \\
97 & 90 \\
64 & 54 \\
116 & 122 \\
99 & 91 \\
62 & 57 \\
\hline
\end{tabular}

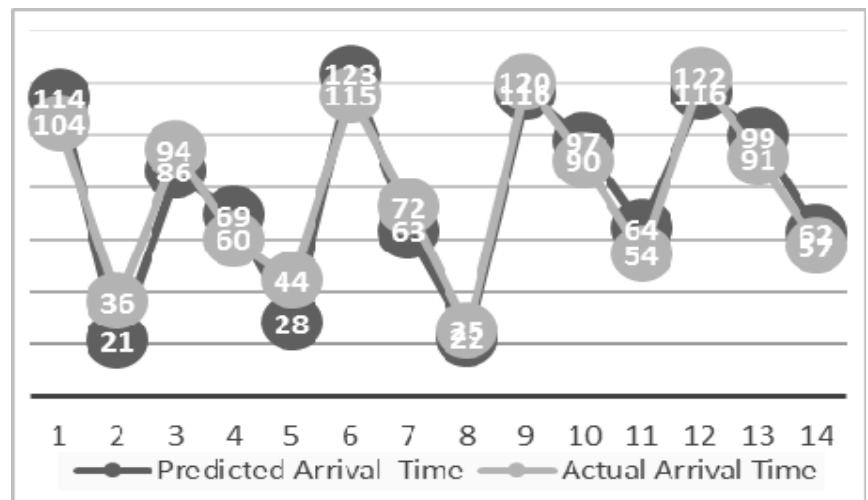

Fig.2. Comparison of the Predicted Arrival Time and Actual Arrival Time

\section{The analysis of experiment results}

The experimental results show that the average difference between the predicted time and the actual time is within the acceptable range. And can be found from the table, the farther you are from the campus bus, the more accurate the prediction of arrival time will be. It can also be seen from the data, with the update of historical data, the prediction is more accurate, which is also consistent with the feature of the algorithm which will be more accurate based on historical data .

\section{CONCLUSION}

In this paper, a method for predicting the arrival time of campus bus is introduced. According to the characteristics of small traffic jam, this paper adopts the prediction model based on historical average data. The accuracy of prediction is improved by dividing the route. The historical data is updated by exponential smoothing method, which makes the historical data more real-time. The accuracy and feasibility of the model are verified by experiments.

\section{REFERENCES}

[1] JI.Xiaoxi.Bus Arrival Time Prediction Model and Empirical Study [D].Beijing: Beijing Jiaotong University.2015

[2] ZHANG.Zhiwei.Design of Bus Arrival Time Prediction Algorithm Based on Macnine Learning[D].Beijing: Beijing University of Post and Telecommunication.2014 